\title{
Abrasive Disc Performance in Dry-Cutting of Medium-Carbon Steel
}

\author{
Naiara Ortega ${ }^{1, *}$, Vitaliy Martynenko ${ }^{2,3}$, Daniela Perez ${ }^{2,3}$, Daniel Martinez Krahmer ${ }^{2,3}$, \\ Luis Norberto López de Lacalle ${ }^{1, *(1)}$ and Eneko Ukar ${ }^{1}$ \\ 1 Department of Mechanical Engineering, Aeronautics Advanced Manufacturing Center (CFAA), Faculty of \\ Engineering of Bilbao, Alameda de Urquijo s/n, 48013 Bilbao, Spain; eneko.ukar@ehu.eus \\ 2 Center for Research and Development in Mechanics, National Institute of Industrial Technology (INTI), \\ Avenida General Paz 5445, Miguelete 1650, Provincia de Buenos Aires, Argentina; \\ vmartynenko@inti.gob.ar (V.M.); djperez@inti.gob.ar (D.P.); dmartinez@inti.gob.ar (D.M.K.) \\ 3 Faculty of Engineering, Universidad Nacional de Lomas de Zamora, Juan XXIII y Camino de Cintura, \\ Buenos Aires 1832, Argentina \\ * Correspondence: naiara.ortega@ehu.eus (N.O.); norberto.lzlacalle@ehu.eus (L.N.L.d.L.); \\ Tel.: +34-946014216 (N.O. \& L.N.L.d.L.)
}

Received: 22 March 2020; Accepted: 19 April 2020; Published: 21 April 2020

\begin{abstract}
Abrasive-cutting processes are widely used to obtain semi-finished products from metal bars, slabs, or tubes. Thus, the abrasive cutting-off process is applied when requiring precision cutting and productivity at a moderate price. Cut-off tools are discs composed of small abrasive particles embedded in a bonding material, called the binder. This work aims to compare the cutting performance of discs with different composition, in dry cutting of steel bars. To do that, disc wear was measured and disc final topography was digitalized in order to determine both disc surface wear patterns and if the abrasive particles bonding into the binder matrix was affected. In addition, X-Ray inspection gave information about the abrasive grit-binder bonding. Therefore, the method here presented allows identifying discs with a superior abrasive-cutting capability, by combining profilometry and tomography to define micrometrical aspects, grit size, and binder matrix structure. Results led to the conclusion that discs with high grit size and protrusion, high grit retention by bond material, and closer mesh of fiberglass matrix binder were the optimal solution.
\end{abstract}

Keywords: dry-cutting; abrasive-cutting; cutting disc; wear; cut-off abrasive wheel

\section{Introduction}

Abrasive discs are commonly used in metalworking workshops to cut off slabs and rods of low and medium carbon steels. This type of tool allows cutting, trimming, deburring, welding blank preparation, and surface polishing. For machinists and technicians, disc cost and service life are important aspects in daily production. On the other hand, (a) abrasive type and size and (b) disc binder are the two most influencing variables. Taking this into account, there are two abrasive disc types for manual machines, (a) those with metal body that use diamond as abrasive, typically used for cutting masonry, marble, and ceramics [1,2], and (b) those usually including aluminum oxide $\left(\mathrm{Al}_{2} \mathrm{O}_{3}\right)$ or silicon carbide $\left(\mathrm{Si}_{3} \mathrm{~N}_{4}\right)$ as grit materials, commonly used for cutting metals [3,4].

Abrasive cutting is basically an operation producing a narrow and deep groove by abrasion till reaching the entire piece cut off. For instance, in the work presented here, abrasive discs are used in cutting rectangular rolled profiles of structural steel. This is an operation typically performed in dry conditions since the use of coolants using manual or semiautomatic machines is always complicated. 
The abrasive-cutting process was widely studied by Neugebauer et al. [4], who analyzed the influence of the $\mathrm{Al}_{2} \mathrm{O}_{3}$ abrasive particle shape on the number of cuts per disc; here the wear criterion was reduction of disc diameter of $25 \mathrm{~mm}$, analyzing three types of geometries on cutting tubes of different materials and diameters. Results showed that those discs with cubic grains produced at least eight percent longer cutting length. However, wear mechanisms were not described in depth.

In addition, Bianchi et al. [5] evaluated the incidence of binder hardness on cutting force and the G-ratio (volume of material removed per unit of volume of wheel wear) using abrasive discs with three different hardness values, finding a light increase of cutting force with hardness, whereas there were no differences with respect to G-ratio; the little increase in cutting force should have needed further experiments.

In grinding, it is well-known the operation of dressing and recovering the wheel shape. Wheel dressing is required when (a) either a form-grinding wheel must keep its shape or (b) when wheel binder hardness is so high that it does not allow the just worn abrasive particles to be released. Therefore, dressing is required to return wheel shape back to its original geometry and/or cutting ability. Thus, Chowdhury et al. [6] developed a method for wheel wear evaluation by means of images taken from wheel topography, under different dressing conditions using rotary dressing tools. Moreover, Daneshi et al. [7] worked on rotary dressing of Cubic Boron Nitride (CBN) and corundum grinding wheels. They found out that up-dressing, in comparison to down-dressing, significantly improved roughness in ground parts. However, their conclusions only applied to the corundum wheel case, since CBN wheels did not suffer quantifiable wear $(<1 \mu \mathrm{m})$.

In addition, Palmer et al. [8] carried out a complete work on diamond dressing of two types of grinding wheels, one with engineered grit (special and defined pattern) and the other with random grit, both aimed at grinding nickel super alloys. Thus, they studied the influence of feed and dressing speed in process performance. They measured power and roughness on ground surfaces on one hand, and abrasive wheels topography by SEM on the other; the main conclusion was that feed speed of the diamond dresser is the most influencing parameter, concluding that when feed increases, power consumption simultaneously reduces, but simultaneously roughness also increases. For engineered grits this relationship did not seem so clear.

Lagel et al. [9] compared discs with eco-resins as binder with traditional ones. These eco-disks included three different abrasive average grit sizes: $0.7,0.4$, and $0.25 \mathrm{~mm}$. Eco-disc with grit size $0.25 \mathrm{~mm}$ and $150 \%$ proportion of abrasive material/binder showed the best performance. Baidakova and Orlova [10] focused on evaluating the influence of form and homogeneity of abrasive particles on cutting performance; grinding wheels with engineered grit offered good results since they reduced cutting time, improved workpiece roughness, and lasted $20 \%$ and $25 \%$ longer. Ni et al. [11] performed cutting tests using four different lubri-cooling techniques, all of them environmentally friendly (dry; water; water + graphite; water + surfactant; water + graphite + surfactant). Through simultaneous measurement of G-ratio, shear force, and cut surface inclination, the best results corresponded in the case water + surfactant. However, no relationship was established between lubrication fluids physical properties and obtained results.

One abrasive wheel degradation type is usually known as "clogging". Rodriguez et al. [12] experimented with different lubrication systems (conventional, MQL minimum quantity of lubricant and MQL + cleaning jet), in the external cylindrical grinding of a steel AISI 4340 taking clogging as main degradation process indicator. They concluded that the proposed MQL + cleaning jet system used $75 \%$ less cutting fluid but slightly higher adhesion appeared. However, AISI 4340 steel did not seem to cause clogging on abrasive wheels.

Denkena and Grove [13] studied the interdependencies between bronze bonded diamond grinding wheels during hard metal grinding. Results showed that both grinding forces (normal and tangential) as well as abrasive wheel wear decreased. However, the influence of cutting temperature on wheel wear was not addressed. 
Zhao et al. [14] performed single-grain grinding tests of a titanium alloy with a conventional CBN and another with modified abrasive grains. They recorded grinding forces, G-ratio, and wear. The main conclusion was that the greater number of cutting edges available in the modified CBN grain made the process enhanced.

The present work aims at evaluating the cutting performance of abrasive discs in the dry-cutting of medium-carbon steel bars. The "stop-cutting" testing criterion was fixed based on the total surface of steel to cut, while other authors used cutting length [4]. Likewise, disc wear was measured by (a) the variation of wheel external diameter and (b) the disc weight loss. Moreover, final abrasive disc profile and fine topography were evaluated to determine the abrasive particles bonding into the matrix binder.

\section{Materials and Methods}

Tested material was hot-rolled rectangular profiles of a mild steel (steel AISI 1020, U.T.S. 424 MPa, hardness $120 \mathrm{HBN}$ ) cross-section of $32 \mathrm{~mm} \times 8 \mathrm{~mm}$. Steel AISI 1020 was (C $0.21 \mathrm{wt} \%, \mathrm{Mn} 0.42 \mathrm{wt} \%$ ), very common in structural applications and also used as profiles for light machinery construction. An "in house" test bench was specially designed and adapted for testing in this project; it was very stiff to eliminate vibrations and geometrical inaccuracies. The testing bench (Figure 1) consisted in a table on which the rolled profiles were clamped by jaws. Working table was able to move in the horizontal plane in two axes by means of two DC servomotors, regulated by a programmable control.
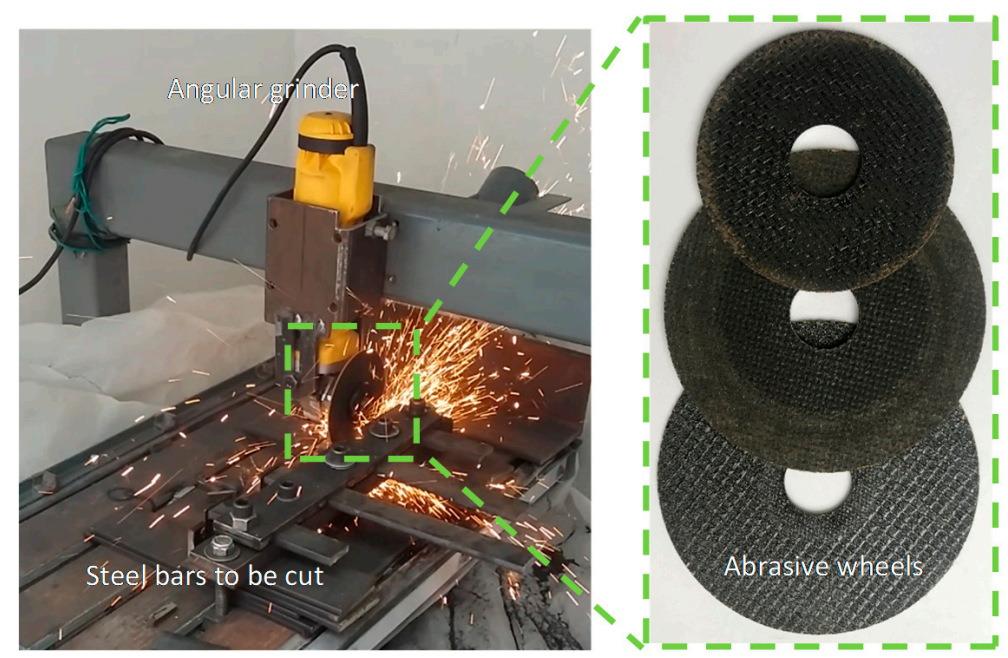

Figure 1. Set up used for experimental tests: manual grinder on a stiff bridge structure. Working table includes two movements, $\mathrm{X}$ and $\mathrm{Y}$ onto rectangular profile is clamped.

Regarding the main spindle, it was a manual angle grinder (Dewalt ${ }^{\mathrm{TM}}$ model DWE 4214, Leola, PA, USA) of power $1200 \mathrm{~W}$ and 11,000 rpm, able to move abrasive-cutting discs of $\Phi 115$ mm maximum. This headstock was fixed onto a structural bridge located above the table, provided with manual height adjustment to set the position between discs and test piece. All tests were carried out under dry conditions to speed up the wear process, and in that way make feasible lab testing without huge amount of test pieces; on the other hand, dry is a typical way of cutting in workshops. Cut-off operations are frequently performed in-situ, where the use of coolants or minimum quantity of lubricant is not easy.

Some preliminary tests were performed to define the maximum feed rate taking into account the maximum of 7 A electrical current provided by the grinder. This resulted in a speed of $250 \mathrm{~mm} / \mathrm{min}$, being kept constant in all the tests performed. As the "end-of-test criterion", a quantity of 12 cuts per disc was established, which is equivalent to a total cut surface of $3072 \mathrm{~mm}^{2}$ per experiment, in order to make wear measurement more independent of both steel profile and disc diameter (disc diameter changes during tests because of wear). A total of sixty-eight different disc types of 17 different compositions were tested. Finally, four types that showed wear levels with significant differences were studied in detail in this work: 
- those with the highest wear, named DE;

- those with the lowest wear, named PE and SP; and

- $\quad$ those with intermediate wear, named TB.

With each disc type, four cutting repetitions were done; maximum divergence allowed was $5 \%$ before repeating one irregular test.

PE, SP, and TB had a grain size \#46, which corresponds to an average diameter of $0.355 \mathrm{~mm}$ and different binder materials. Disc thickness ranged between $1.60 \mathrm{~mm}$ and $1.65 \mathrm{~mm}$. On the other hand, DE disc type had grain size of $0.250 \mathrm{~mm}$ and thickness of $1.50 \mathrm{~mm}$.

After cutting tests, macrogeometric disc wear was measured using different techniques, namely, (i) a caliper, (ii) by disc weight change just before and after each test, (iii) by means of optical microscopy, and (iv) by confocal microscope. Measurement purpose was to estimate discs' mass loss and diametric reduction. Diameter variation was checked using a Tesa $@$ digital caliper model 00530084 (Tesa, Renens, Switzerland). For weight variation, Moretti@ electronic scale, model OAC 2.4 with 0.2 resolution was used. Discs measurements were performed before (new) and after (final) the tests.

Subsequently, disc surfaces were digitalized to evaluate the loss of abrasive-cutting ability (microgeometric wear). Thus, Leica@ DMS300 optical microscope and Leica DCM30 confocal microscope (Leica, Wetzlar, Germany) were used for that purpose. Standard ISO 25178 roughness parameters were used to quantify microgeometric wear.

Surface roughness of steel specimens was measured with a portable profilometer (Taylor Hobson ${ }^{\circledR}$ model Surtronic 3+, Leicester, UK) and cut surface images were taken by scanning electron microscopy (FEI ${ }^{\mathrm{TM}}$ model QUANTA 250 FEG, Hillsboro, OR, USA). Finally, discs were analyzed by computerized tomography by means of X-Cube A590520 (Seifert X-ray ${ }^{\circledR}$, Fairview Village, PA, USA) compact $225 \mathrm{kV}$ from GEC.

\section{Results and Discussion}

Experimental results regarding different aspects are explained in the following sections, those are: macrogeometry wear, microgeometric wear, and final cut surface quality.

\subsection{Macrogeometric Wear}

Macrogeometric wear is defined as disc diameter loss or reduction. This wear type follows a typical wear curve, similar to several abrasion degradation processes. Results are gathered in Table 1 , which shows average variation in diameter and weight of the four disc types analyzed. Average thickness of each disc type, material removed, and the calculated G-ratio are also indicated.

Table 1. Diametric wear, weight wear and G-Ratio results (average values).

\begin{tabular}{lccccccccc}
\hline $\begin{array}{l}\text { Disc } \\
\text { Ref. }\end{array}$ & $\begin{array}{c}\text { Initial } \\
\mathbf{( m m})\end{array}$ & $\begin{array}{c}\mathbf{\Phi} \text { Final } \\
\mathbf{( m m})\end{array}$ & $\begin{array}{c}\mathbf{\Phi} \\
\text { Variation } \\
\mathbf{( m m})\end{array}$ & $\begin{array}{c}\text { Initial } \\
\text { Weight } \\
\mathbf{( g )}\end{array}$ & $\begin{array}{c}\text { Final } \\
\text { Weight } \\
\mathbf{( g )}\end{array}$ & $\begin{array}{c}\text { Variation } \\
\mathbf{( g )}\end{array}$ & $\begin{array}{c}\text { Thickness } \\
\mathbf{( m m})\end{array}$ & $\begin{array}{c}\text { Volume } \\
\text { Removed } \\
\left(\mathbf{m m}^{3}\right)\end{array}$ & G-Ratio \\
\hline $\mathrm{DE}$ & 115.25 & 83.83 & 31.42 & 34.80 & 17.80 & 17.00 & 1.50 & 4608.0 & 0.63 \\
$\mathrm{PE}$ & 115.45 & 109.67 & 5.78 & 37.60 & 33.20 & 4.40 & 1.65 & 5068.8 & 3.01 \\
$\mathrm{SP}$ & 115.18 & 109.33 & 5.85 & 37.20 & 32.80 & 4.40 & 1.60 & 4915.2 & 2.98 \\
$\mathrm{~TB}$ & 115.37 & 105.24 & 10.13 & 39.80 & 32.20 & 7.60 & 1.65 & 5068.8 & 1.75 \\
\hline
\end{tabular}

After 12 cuts per disc, results showed that PE and TB discs removed the highest volume, followed by SP and DE discs. In contrast with [5], wear indicator G-ratio showed noticeable differences. The highest material removal ability relative to wear was PE disc followed by SP disc. 
Figure 2 presents wear measured per millimeter of disc width. The results show a linear correlation which gives an idea of the reliability of the results obtained. The general trend is similar to that presented by Martynenko et al. [2] for cutting concrete.

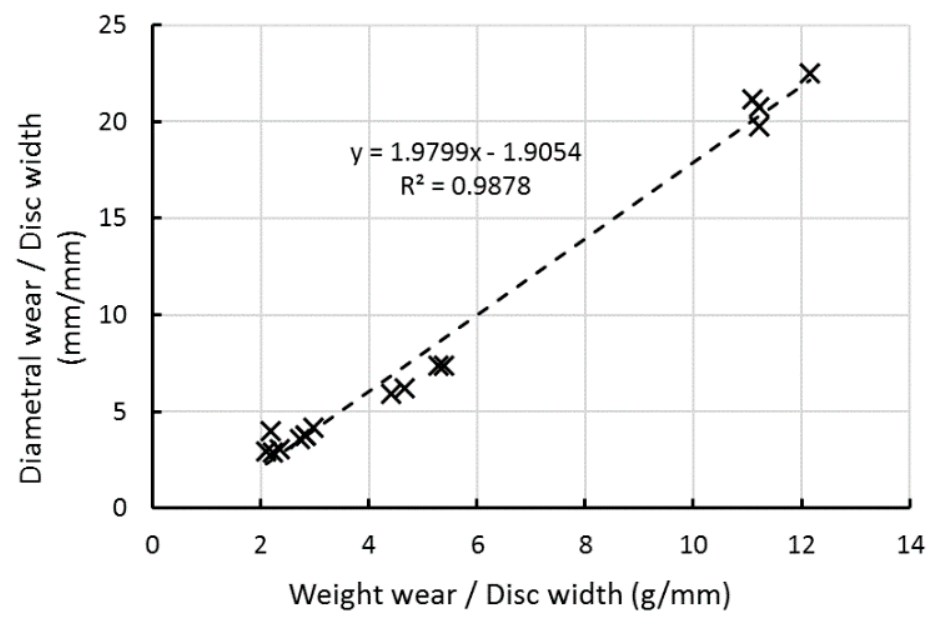

Figure 2. Correlation between radial wear and disc weight loss per disc width.

Measurements performed by confocal microscope obtaining wear along disc cross section, which in some cases turned out to be non-homogeneous. Hence, Figure 3 shows four 3D topographies obtained from one from each of the four disc types. Regarding disc DE and TB, a more homogenous texture is observed without distinction between grits and bonding material since grain protrusions are lower. This is, if the length from $4 \mathrm{~mm}$ to $10 \mathrm{~mm}$ of the $X$ axis is taken, the height varies approximately between $1100 \mathrm{~mm}$ and $1300 \mathrm{~mm}$.
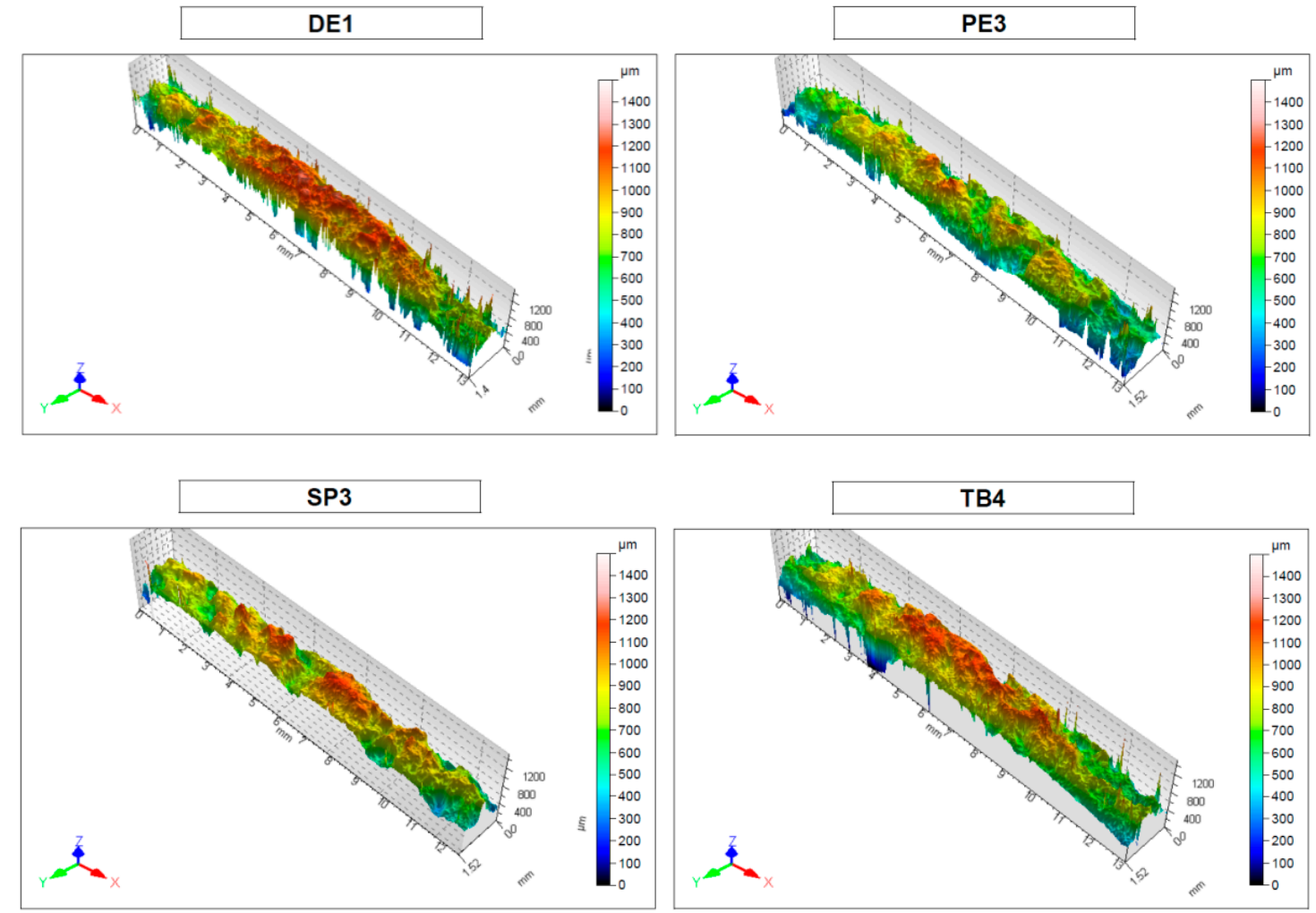

Figure 3. 3D topography of the worn surface of DE1, PE3, SP3, and TB4 discs. 
Thus, from Figure 3, results seem to show that DE and TB discs had the flattest areas, while PE and SP discs show abrasive grains with spacing between them, which implies greater abrasive-cutting and spare room space for removed little chips. Some more specific measurements are in Figure 4.
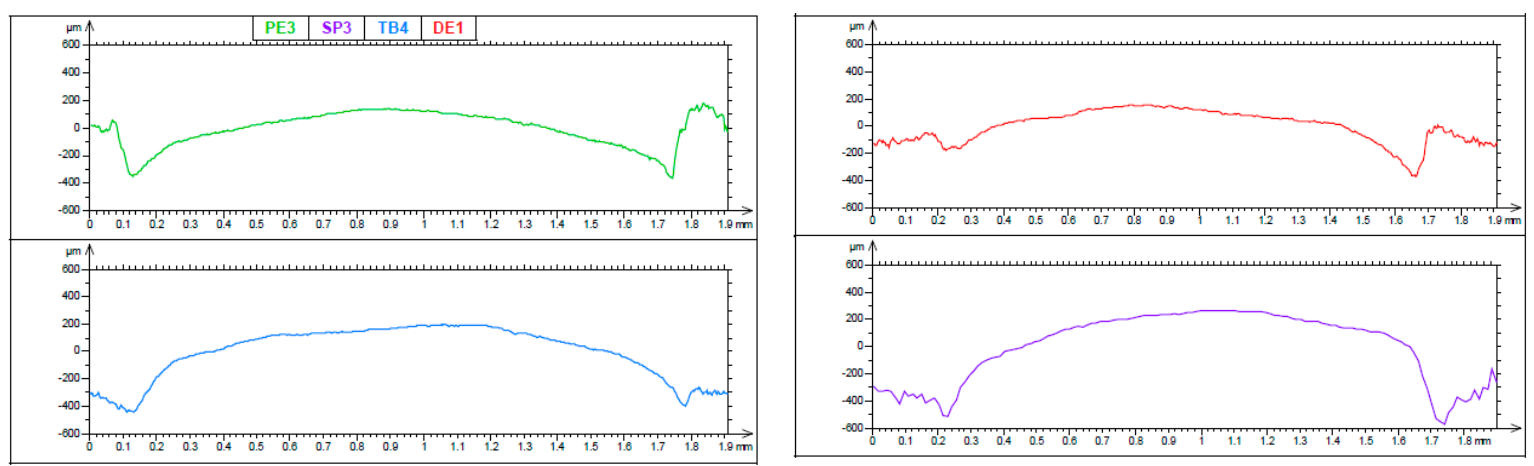

Figure 4. Cross sections of worn discs: upper row PE and DE, lower row TB and SP.

In Figure 4, profiles that determine the discs form degradation are shown. Results indicate that $\mathrm{DE}$ disc is the one losing material uniformly over its entire width, with the flattest profile. In the rest of the discs, however, a more pronounced wear appears on the sides, with the SP disc presenting a lower radius in the profile (less uniform wear). This wear is closely related to the grade of the disc, and is essential to prevent damage to the bar cut surface. The profile flattening shape implies a larger contact wheel-part surface with higher force [15].

\subsection{Microgeometric Wear}

Firstly, microgeometric wear suffered by each disc were sorted out. Abott-Firestone curves of the 3D digitalized surface were analyzed, as they are one of the graphs that in roughness measurements do not use a mean value line, which is interesting for this type of non-homogeneous surface.

The Abott-Firestone curve functional parameters (based on graphical construction) are Sk, Spk, and Svk. Thus, $S k$ is the "thickness" of the surface central part, this is, the core; Spk is the peak height above the core; and Svk is the valley depth below the core. Taking these definitions into account, Spk seems to be the most representative parameter to quantify the discs surface wear since it is an indicator of surface flatness compared to core and valleys.

In Figure 5, the highest value for Spk corresponds to PE disc $(S p k=0.171 \mu \mathrm{m})$, as this is the disc with lower wear, and the lowest value $(S p k=0.092 \mu \mathrm{m})$ that corresponds to the TB as the most worn disc. As result, this $3 \mathrm{D}$ roughness parameter could be used as an indicator for the abrasive-cutting ability of the disc.

The average peak height value in a disc section was measured to know the grain abrasive-cutting ability. The results are shown in Figure 6 where SP disc appears as the one with the highest cutting ability (average grain exposure $=0.23 \mathrm{~mm}$ ), and has the highest grain exposure or protrusion. PE and TB discs have a medium height value of exposed grains. DE discs have the least exposure, which is consistent with a smaller size (average grain exposure $=0.088 \mathrm{~mm}$ ).

Finally, the discs were analyzed by means of X-rays (computed tomography) to observe their internal composition, and by optical stereo microscope (see Figure 7). The thin abrasive-cutting discs are reinforced with fiberglass, nylon, carbon, cotton, linen, wood, silk, or other materials [16]. Reinforcement structures can follow various patterns. In the discs under this study, reinforcement is made of fiberglass with a flat pattern. However, there were great differences between them, because they come from different manufacturers. Figure 7 show the different structures of DE and PE discs using a Leica DMS300 microscope at 10×. This was measured to quantify the different surface patterns. 

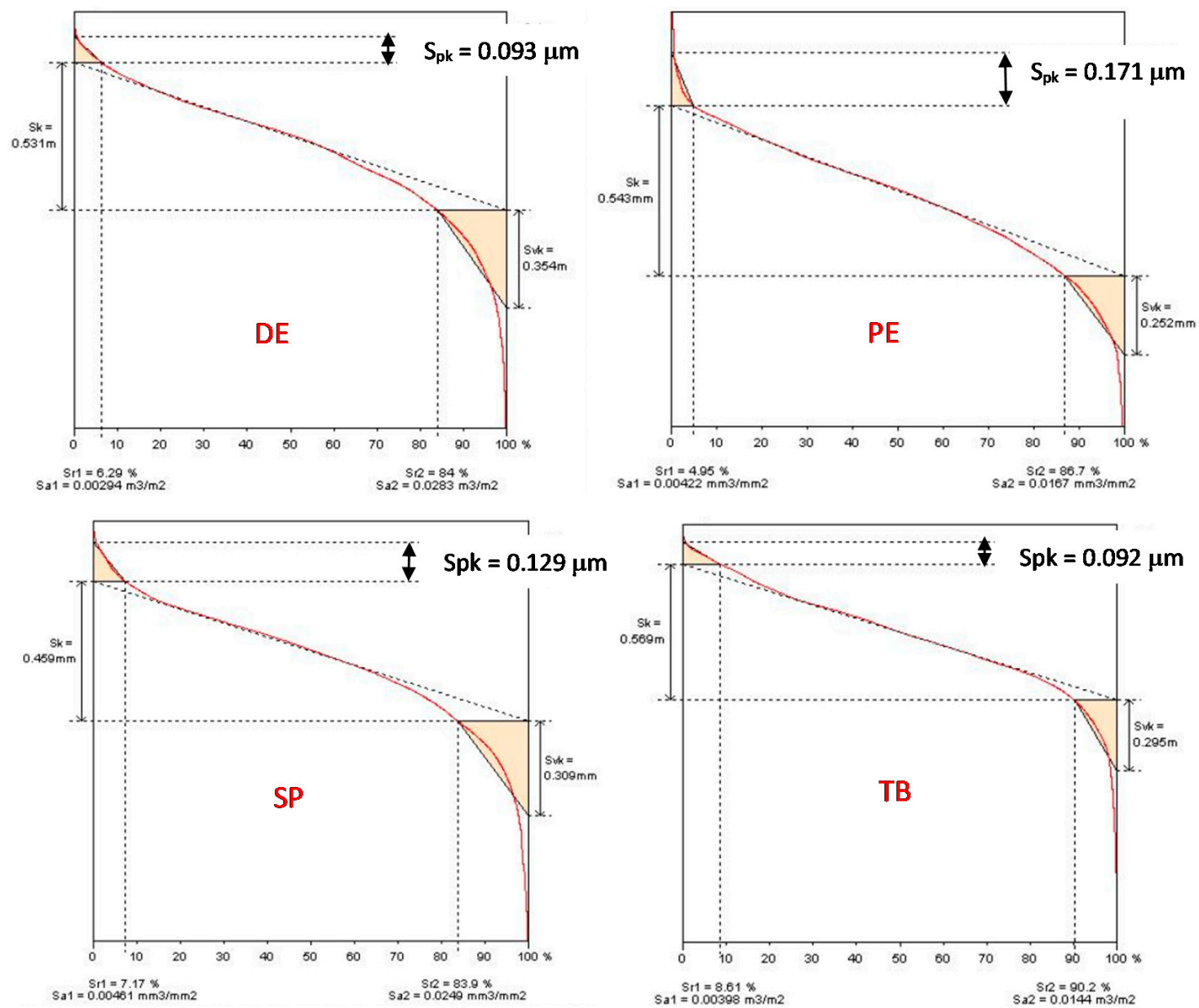

Figure 5. Abott-Firestone curves of worn discs.
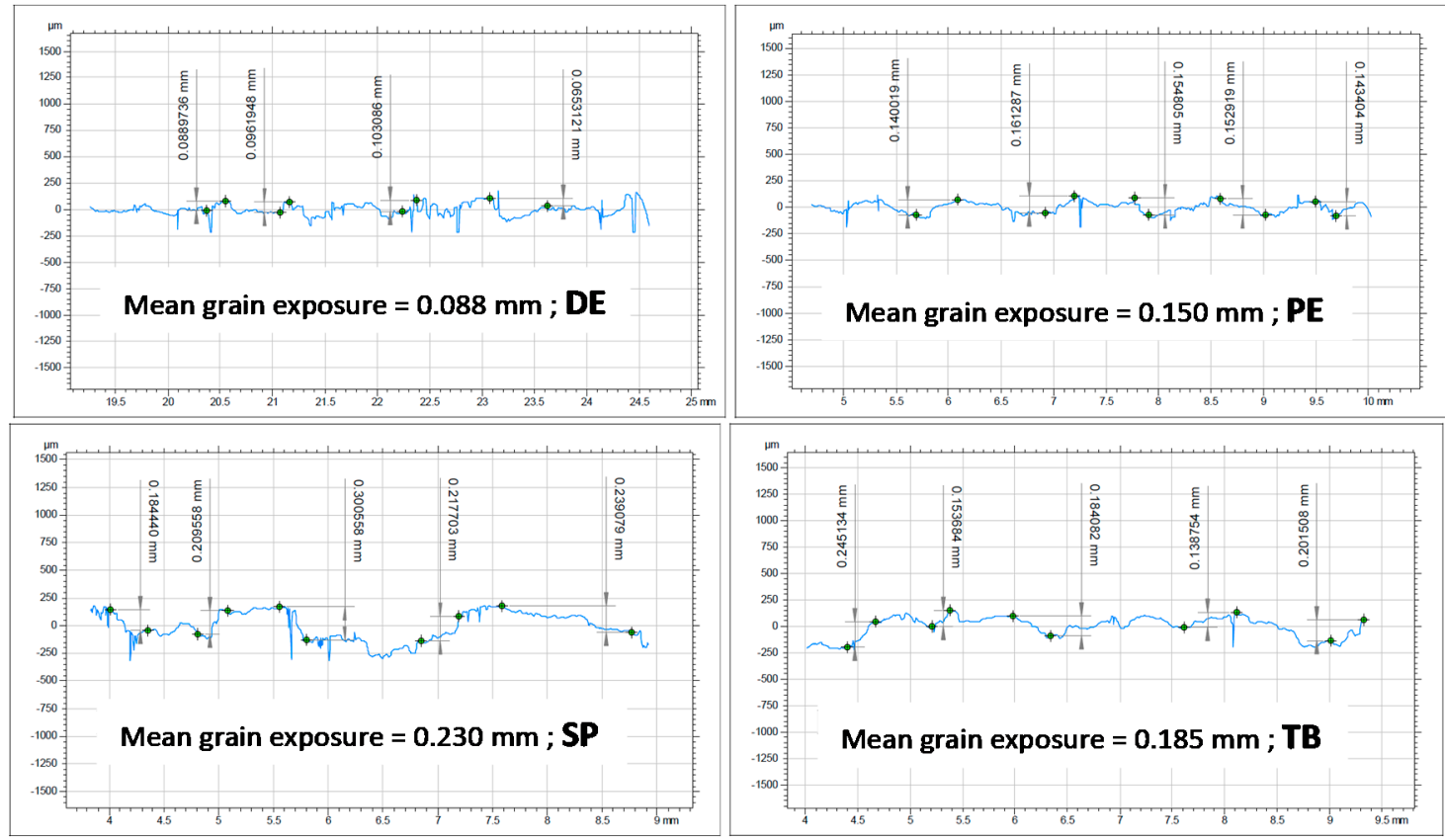

Figure 6. Measurement of the average grain height of worn discs. 

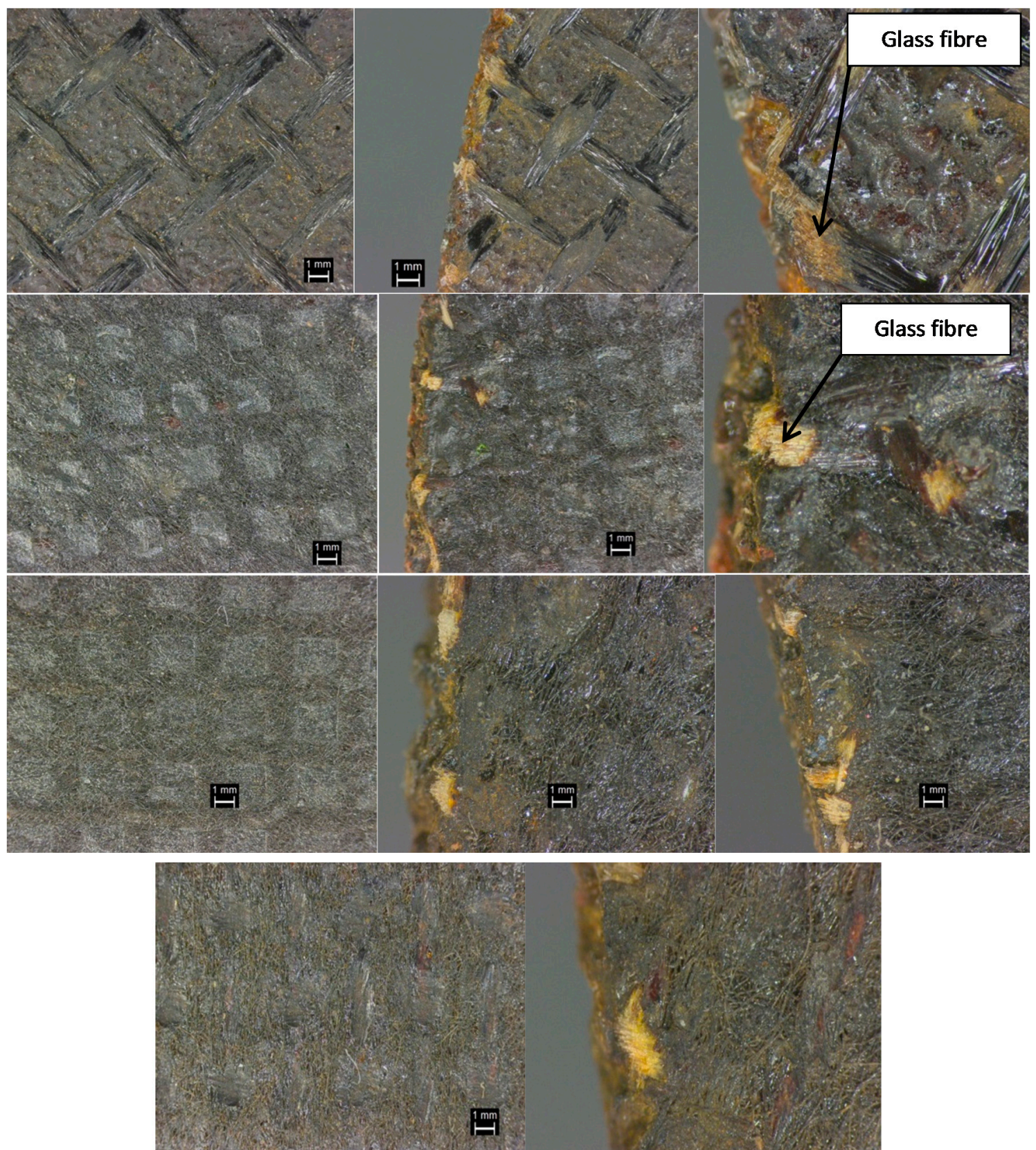

Figure 7. From top to bottom: (1st row) XR picture of the structure of DE disc, (2nd row) XR picture of the structure of PE disc, (3rd row) XR picture of the structure of SP disc, (4th row) XR picture of the TB disc structure.

Thus, DE disc has a wider structure with an inner square side of $1.75 \mathrm{~mm}$ and a fiberglass width of $0.62 \mathrm{~mm}$. PE disc, on the other hand, has side dimensions of $1.00 \mathrm{~mm}$ with a fiberglass width of $0.90 \mathrm{~mm}$. SP disc measures $1.16 \mathrm{~mm}$ with a fiberglass width of $0.85 \mathrm{~mm}$ and TB disc measures $1.36 \mathrm{~mm}$ mesh opening side with a fiberglass width of $0.85 \mathrm{~mm}$ (values are the average of 20 measurements per disc).

Thus, the SP disc has a more diffuse structure, even though the geometry is similar to that of the PE disc. The TB disc, however, shows a less flat structure.

From these images and cutting tests, it is concluded that the highest wear is in discs with an large "open mesh" with less fiberglass width, which gives it less resistance to radial wear (macro wear). The rest of the discs showed a similar relationship being the disc with lowest wear was the one with the densest mesh. 
Figure 8 shows the relationship between G-ratio and open mesh size, concluding that mesh size is also another parameter to take into account for disc wear analysis, due to its influence on disc stiffness.

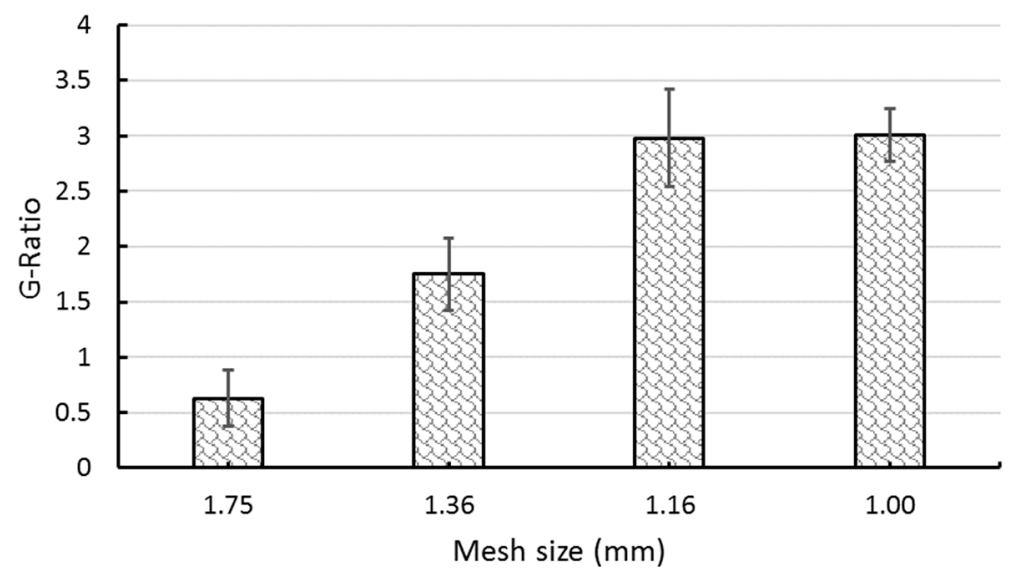

Figure 8. Relationship between G-ratio and mesh opening, average value, and deviation.

Regarding X-ray inspection, it indicates that all the discs had the same density in all their volume from the outside to the inside (each one), although there were differences in density between them. Figure 9 shows the difference. Thus, in DE discs there were larger areas of lower density than in PE discs, that is to say, the retention of the mesh is smaller in DE discs, which increases due to the smaller grain size. This is another reason why DE discs had greater wear than the other three types, which have a more homogeneous density. Some literature observations are related with failure in those reinforced by woven roving type glass fiber [16,17].

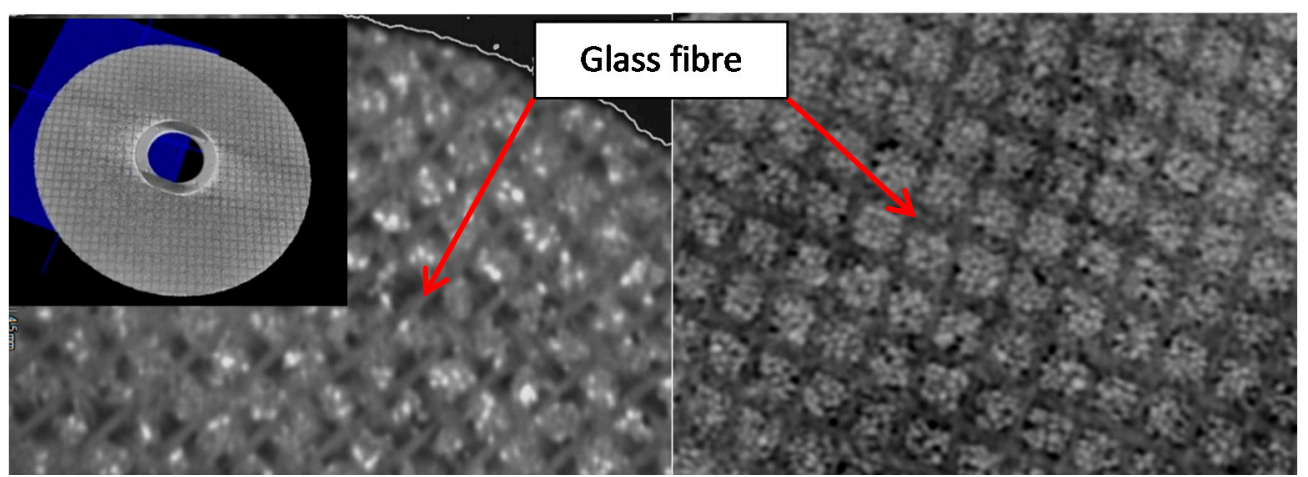

Figure 9. X-ray image of DE disc (shown top left corner) and PE disc.

\subsection{Part Quality}

To study whether the part was affected by disc degradation and quality, some surfaces of the trimmed steel profiles were analyzed, in the cases of discs with the greatest wear (i.e., DE-sample DE1) and the one with the lowest wear (PE-sample PE3).

For this purpose, roughness of cut surfaces was measured and images were obtained by means of SEM. Roughness results are shown in Table 2.

Table 2. Results of roughness in pieces cut with discs DE1 and PE3.

\begin{tabular}{ccc}
\hline Sample & Average Ra $(\mu \mathrm{m})$ & Standard Deviation $(\mu \mathrm{m})$ \\
\hline DE1 & 2.09 & \pm 0.56 \\
PE3 & 1.40 & \pm 0.36 \\
\hline
\end{tabular}


As shown in Table 2, roughness after abrasive-cutting with DE disc is $49 \%$ greater than with the PE disc.

On the one hand, the grain retained in a firmer way causes the grain to wear flat, thus producing highly negative rake angles at the microgeometry of cutting (abrasive grains produce a micro removal material cutting process, so rake angle can be defined as in the machining case). This type of wear increases power consumption and can damage the steel part in severe cases, but at the same time improve the surface finish by predominating sloughing and rubbing effects over shearing (shearing is the most important process in cutting). On the other hand, the softer disc, with higher wear, has a binder that retains abrasive grains with less force, thus releasing them instead of having heavy grain wear. Consequently, its abrasive-cutting ability is more aggressive, leaving higher roughness in cut surfaces. In addition, although this type of disc generates a higher consumption of wheel, it usually has lower power consumption [18]. Figure 10 shows SEM images corresponding to the bar cut surfaces with both DE and PE discs.
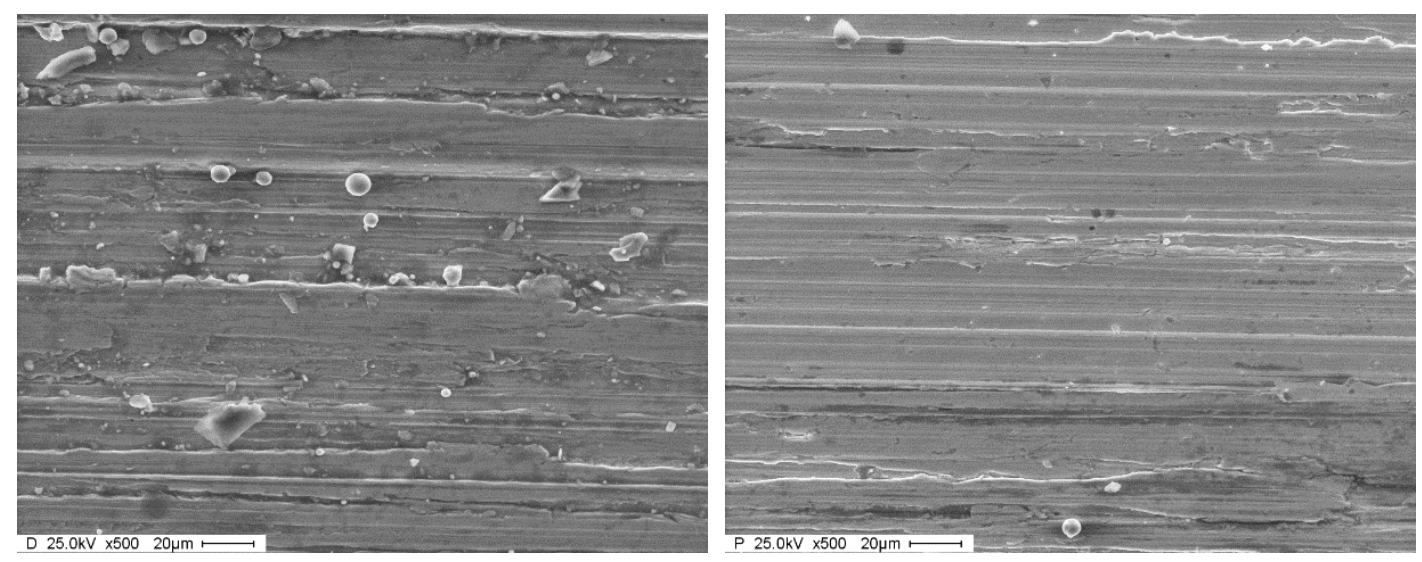

Figure 10. SEM images of cut surface with DE1 disc (left) and PE3 disc (right).

Hence, on the surface cut by DE disc (soft disc), a more irregular surface pattern appeared, with some areas including adhered particles. The particles belonged to the steel bar itself, which were not properly evacuated as no coolant was used, while other particles came from abrasive disc grains that were detached during the abrasive process.

Surfaces corresponding to PE disc cutting (harder disc) presented a more uniform aspect almost without adhesion, so in this case, piece surface quality was better. Although metallographic studies were also carried out in order to determine possible microstructural changes (thermally affected zone, grain size, microhardness), no differences were observed during these studies.

Finally, Table 3 evaluates the cutting discs during the tests, rated from 1 (best) to 4 (worst), so that the lowest summation case will be globally the best and the one with the highest summation will be globally the worst.

In this way, the discs are ranked PE, SP, TB, and DE from the best to the worst in overall performance. Regarding prices, there is a not significant difference among them.

Table 3. Numerical qualification of the various discs tested, for the characteristics evaluated.

\begin{tabular}{cccccccc}
\hline Type & $\boldsymbol{\Delta} \boldsymbol{\Phi}$ & $\boldsymbol{\Delta} \mathbf{P}$ & G-Ratio & $\boldsymbol{S p k}$ & Grain Exposure & Mesh Size & Total \\
\hline DE & 4 & 3 & 4 & 3 & 4 & 4 & 22 \\
PE & 1 & 1 & 1 & 1 & 3 & 1 & 8 \\
SP & 2 & 1 & 2 & 2 & 1 & 2 & 10 \\
TB & 3 & 2 & 3 & 4 & 2 & 3 & 17 \\
\hline
\end{tabular}

Note: $\Delta \Phi$ is the diameter loss and $\Delta \mathrm{P}$ the weight loss. 


\section{Conclusions}

In this work the wear analysis of discs used for abrasive-cutting of mild steel bars has been carried out.

- $\quad$ Four disc types with different compositions have been studied. PE disc offers the highest cutting capacity with minimum wear (greater G-ratio).

- Disc wear has been measured macro and micro geometrically. The decrease in diameter has been evaluated in two ways, by weigh control and by measuring the diameter with a caliper, while the loss of abrasive-cutting ability has been analyzed by 3D functional parameters of the Abott-Firestone curve (mainly $S p k$ ) and by 2D evaluation of the cut surface.

- In relation with the internal mesh abrasive structure, is concluded that, discs with the greatest wear were those with a more "open mesh" structure. Also, a linear relationship has been established between open mesh quality and G-ratio for the studied discs.

- $\quad$ By means of X-rays, internal density of discs binder has been evaluated, concluding that discs with the highest wear (DE) has low density, which increases its wear due to lack of resistance.

- Abrasive disc wheels were defined by four parameters: disc-surface contact flatness, grain exposure, parameter $S p k$, and mesh size.

In summary, abrasive discs for mild steel parts should fulfill:

- Higher grit size with high grit protrusion

- Higher grit retention by bond material

- Closer mesh of fiberglass matrix binder

In future work, a load cell will be installed so that the cutting forces will be recorded when dry-cutting with the aim of gathering the power consumption. Changes in grain forms will be tested as well [19]. It is expected that, as the disc wears, the power consumption will increase. However, it should be very interesting to know how the power curve varies as a function of the wear stage so that the disc shelf-sharpening ability can be analyzed. Dissimilar materials cutting would be a next step [20], because tubes and metal profiles are regularly being cut-off by manual sawing machines.

Author Contributions: Conceptualization, D.M.K. and N.O.; methodology, D.P., L.N.L.d.L. and V.M.; investigation and analysis, D.M.K., D.P., N.O., L.N.L.d.L., and V.M.; methodology D.M.K. and N.O.; data curation, E.U.; general view, L.N.L.d.L. All authors have read and agreed to the published version of the manuscript.

Funding: Authors are grateful to Basque government group IT IT1337-19 and Ministry of Mineco REF DPI2016-74845-R.

Acknowledgments: Thanks are addressed to all people staying patiently at home in this virus crisis and all healthcare professionals who take care of other people.

Conflicts of Interest: The authors declare no conflict of interest.

\section{References}

1. Egea, A.J.S.; Martynenko, V.; Krahmer, D.M.; De Lacalle, L.N.L.; Benítez, A.; Genovese, G. On the cutting performance of segmented diamond blades when dry-cutting concrete. Materials 2018, 11, 264. [CrossRef] [PubMed]

2. Martynenko, V.; Antúnez, N.; Benítez, A.; Pérez, D.; Krahmer, D.M.; Genovese, G. Desempeño de discos segmentados de diamante en el corte de hormigón. Rev. Mater. 2018, 23, 2. [CrossRef]

3. Li, H.; Yu, T.B.; Wang, Z.X.; Da Zhu, L.; Wang, W.S. Detailed modeling of cutting forces in grinding process considering variable stages of grain-workpiece micro interactions. Int. J. Mech. Sci. 2017, 126, 319-339. [CrossRef]

4. Neugebauer, R.; Hess, K.-U.; Gleich, S.; Pop, S. Reducing tool wear in abrasive cutting. Int. J. Mach. Tools Manuf. 2005, 45, 1120-1123. [CrossRef] 
5. Bianchi, E.C.; Silva, E.J.; França, T.; Junior, C.S.; Valarelli, I.D. Influência da dureza dos discos abrasivos no corte de materiais dúcteis. Rev. Mater. 2006, 11, 24-29. [CrossRef]

6. Chowdhury, M.; Tamaki, J.; Kubo, A.; Ullah, A.S. Visualization of grinding wheel surface topography for multiple passes of rotary diamond dresser. Adv. Mater. Res. 2012, 565, 222-227. [CrossRef]

7. Daneshi, A.; Jandaghi, N.; Tawakoli, T. Effect of dressing on internal cylindrical grinding. Procedia CIRP 2014, 14, 37-41. [CrossRef]

8. Palmer, J.; Curtis, D.; Novovic, D.; Ghadbeigi, H. The influence of abrasive grit morphology on wheel topography and grinding performance. Procedia CIRP 2017, 77, 239-242. [CrossRef]

9. Lagel, M.C.; Zhang, J.; Pizzi, A. Cutting and grinding wheels for angle grinders with a bioresin matrix. Ind. Crops Prod. 2015, 67, 264-269. [CrossRef]

10. Baidakova, N.; Orlova, T. Usage of abrasive grains with controllable shapes as means of grinding wheels operation stabilization. Procedia Eng. 2017, 206, 188-193. [CrossRef]

11. Ni, J.; Yang, Y.; Wu, C. Assessment of water-based fluids with additives in grinding disc cutting process. J. Clean. Prod. 2019, 212, 593-601. [CrossRef]

12. Rodriguez, R.L.; Lopes, J.C.; Hildebrandt, R.A.; Bianchi, E.C. Evaluation of grinding process using simultaneously MQL technique and cleaning jet on grinding wheel surface. J. Mater. Process. Technol. 2019, 271. [CrossRef]

13. Denkena, B.; Grove, T. Model-based manufacturing and application of metal-bonded grinding wheels. CIRP Ann. Manuf. Technol. 2019, 68, 321-324. [CrossRef]

14. Zhao, B.; Ding, W.F.; Zhou, Y.; Su, H.; Xu, J. Effect of grain wear on material removal behaviour during grinding of Ti-6Al-4V titanium alloy with single aggregated cBN grain. Ceram. Int. 2019. [CrossRef]

15. Turchetta, S. Cutting force in stone machining by diamond disk. Adv. Mater. Sci. Eng. 2010. [CrossRef]

16. Sahu, P.; Sagar, R. Development of abrasive cut-off wheel having side grooves. Int. J. Adv. Manuf. Technol. 2006, 31, 37-40. [CrossRef]

17. Linke, B.S. Life Cycle and Sustainability of Abrasive Tools; Springer International Publishing: Berlin/Heidelberg, Germany, 2016; ISBN 978-3-319-28345-6.

18. Badger, J. Evaluation of triangular, engineered-shape ceramic abrasive in cutting discs. Weld. J. 2014, 93, 107S-115S.

19. Kovalchenko, A.M.; Goel, S.; Zakiev, I.; Pashchenko, E.A.; Al-Sayegh, R. Suppressing scratch-induced brittle fracture in silicon by geometric design modification of the abrasive grits. J. Mater. Res. Technol. 2019, 8, 703-712. [CrossRef]

20. Urbikain, G.; Perez, J.M.; De Lacalle, L.N.L.; Andueza, A.; Urbicain, G. Combination of friction drilling and form tapping processes on dissimilar materials for making nutless joints. Proc. Inst. Mech. Eng. Part B J. Eng. Manuf. 2016, 232, 1007-1020. [CrossRef] 